\title{
Exercise Diminishes Plasma Neurofilament Light Chain and Reroutes the Kynurenine Pathway in Multiple Sclerosis
}

Niklas Joisten, PhD,* Annette Rademacher, ${ }^{*}$ Clemens Warnke, MD, Sebastian Proschinger, Alexander Schenk, PhD, David Walzik, Andre Knoop, PhD, Mario Thevis, PhD, Falk Steffen, Stefan Bittner, MD, Roman Gonzenbach, MD, Jan Kool, PhD, Wilhelm Bloch, MD, Jens Bansi, PhD, † and Philipp Zimmer, PhD, PhD† Neurol Neuroimmunol Neuroinflamm 2021;8:e982. doi:10.1212/NXI.0000000000000982

\section{Abstract}

\section{Objective}

To examine acute (single-bout) and training effects of high-intensity interval training (HIIT) vs standard exercise therapy (moderate continuous training [MCT]) on plasma neurofilament light chain (pNfL) and kynurenine (KYN) pathway of tryptophan degradation metabolites in persons with multiple sclerosis (pwMS).

\section{Methods}

Sixty-nine pwMS (Expanded Disability Status Scale score 3.0-6.0) were randomly assigned to a HIIT or an MCT group. Changes in $\mathrm{pNfL}$ and KYN pathway metabolites measured in blood plasma were assessed before, after, and 3 hours after the first training session as well as after the 3-week training intervention.

\section{Results}

Acute exercise reduced $\mathrm{pNfL}$ and increased the KYN pathway flux toward the neuroprotective kynurenic acid (KA). Changes in pNfL correlated positively with changes in KA and negatively with the quinolinic acid-to-KA ratio. HIIT consistently led to greater effects than MCT. Following the 3-week training intervention, the KYN pathway was activated in HIIT compared with MCT.

\section{Conclusion}

Future studies and clinical assessments of pNfL should consider acute exercise as confounding factor for measurement reliability. Moreover, exercise-induced KYN pathway rerouting might mediate neuroprotection, potentially underlying the benefits in rehabilitation for pwMS.

\section{Classification of Evidence}

This study provides Class II evidence that acute HIIT diminishes pNfL and increases KA levels, and 3 weeks of HIIT activate the KYN pathway in pwMS.

\section{Trial Registration Information}

Clinical trial registration number: NCT03652519.

\author{
Correspondence \\ Dr. Zimmer \\ philipp.zimmer@tu-dortmund.de
}

\section{MORE ONLINE}

$\rightarrow$ Class of Evidence

Criteria for rating

therapeutic and diagnostic

studies

NPub.org/coe

\footnotetext{
*These authors contributed equally to this work.

tThese authors share senior authorship.

From the Department of "Performance and Health (Sports Medicine)" (N.J., A.S., D.W., P.Z.), Institute of Sport and Sport Science, Technical University Dortmund; Department for Molecular and Cellular Sports Medicine (A.R., S.P., W.B.), Institute of Cardiovascular Research and Sports Medicine, German Sport University Cologne; Department of Neurology (C.W.), Faculty of Medicine and University Hospital Cologne, University of Cologne; Center for Preventive Doping Research/Institute of Biochemistry (A.K., M.T.), German Sport University Cologne; Department of Neurology (F.S., S.B.), Focus Program Translational Neuroscience (FTN), and Immunotherapy (FZI), University Medical Center of the Johannes Gutenberg University, Germany; and Department of Neurology (R.G., J.K., J.B.), Clinics of Valens, Rehabilitation Centre Valens, Switzerland. 


\section{Glossary}

CV = coefficient of variation; EDSS = Expanded Disability Status Scale; HIIT = high-intensity interval training; HPLC-MS/ MS = high-performance liquid chromatography-tandem mass spectrometry; IL-6 = interleukin-6; KA = kynurenic acid; KYN = kynurenine; $\mathbf{M C T}=$ moderate continuous training; $\mathbf{p N f L}=$ plasma neurofilament light chain; $\mathbf{p w M S}=$ persons with multiple sclerosis; $\mathbf{Q A}=$ quinolinic acid; TRP = tryptophan.

Plasma neurofilament light chain (pNfL) has become an integrative biomarker of neurodegeneration in persons with multiple sclerosis (pwMS). Higher levels reflect axonal loss and correlate with acute inflammation and disability progression. ${ }^{1}$ The $\mathrm{pNfL}$ level is associated with various disease specific clinical and MRI-related outcomes such as acute relapses, T2 lesion load, or future brain atrophy. ${ }^{2,3}$

The kynurenine (KYN) pathway of tryptophan (TRP) degradation is involved in different neuroinflammatory and neurodegenerative processes. ${ }^{4}$ In pwMS, the KYN pathway is deregulated and has recently attracted extensive attention as therapeutic target. ${ }^{5}$ Persistent overactivations of the KYN pathway are indicated by an elevated KYN/TRP ratio compared with healthy controls. ${ }^{6}$

Recently, 2 studies uncovered a relationship between $\mathrm{NfL}$ and the KYN pathway in the context of neurodegeneration. In a cohort of older adults, pNfL levels were positively associated with several KYN pathway metabolites. ${ }^{7}$ In pwMS, NfL levels correlated positively with the neurotoxic KYN pathway metabolite quinolinic acid (QA) in the CSF. ${ }^{8}$

Physical exercise alleviates various symptoms in pwMS, and disease-modifying effects are discussed. ${ }^{9}$ Especially concerning physical fitness and disease-associated biomarkers, high-intensity interval training (HIIT) leads to greater improvements than classical moderate continuous training (MCT) ${ }^{10-12}$ A better understanding of exercise-induced mechanisms would improve our understanding of disease-modifying effects and help to develop targeted exercise recommendations for pwMS.

Here, we (1) investigate the influence of exercise on pNfL in pwMS and (2) shed light on the association between exerciseinduced alterations of the KYN pathway and $\mathrm{pNfL}$.

\section{Methods}

This study is a secondary analysis of a prospective single-blind randomized controlled trial that was conducted in the inpatient rehabilitation clinic Valens (Switzerland). ${ }^{13}$ The study was approved by the regional ethics committee (EKOS18/96; Project-ID: 2018-01378) and registered at ClinicalTrials.gov (NCT03652519; August 29, 2018). Written informed consent was obtained from all participating patients. Detailed information on eligibility criteria can be depicted elsewhere. ${ }^{13}$ Briefly, the inclusion criteria comprised a definite diagnosis of MS, age $>21$ years, a relapsing-remitting or secondary progressive MS phenotype, and an Expanded Disability Status Scale (EDSS) score between 3 and 6. Main exclusion criteria were concomitant diseases, pregnancy, alcohol or drug abuse, and a second participation in this investigation. In total, 69 pwMS with either relapsing-remitting ( $\mathrm{n}=$ $42)$ or secondary progressive $(n=27)$ phenotype and a mean \pm SD EDSS score of $4.51 \pm 1.06$ were included. Participant baseline characteristics are shown in table 1. Baseline cognitive performance of participants was assessed using the Brief International Cognitive Assessment in Multiple Sclerosis ${ }^{14}$ to verify an association between baseline pNfL levels and a clinical outcome within our sample. The assessment of cognitive performance was conducted within 24 hours after the baseline blood sample collection.

Participants were randomized with concealed allocation in either a HIIT or a MCT (standard therapy) group using physical fitness, EDSS score, age, and fatigue as stratification factors.

\section{Groups and Interventions}

Participants of both groups conducted a 3-week training intervention during inpatient rehabilitation with 3 exercise sessions per week. The individual exercise intensity was calculated based on the maximum heart rate achieved in an incremental exercise test until symptom-limited exhaustion before the intervention. Each session in both groups included a warm-up and cool-down period of 3 minutes. Although the participants of the MCT group exercised continuously for 24 minutes at an intensity of $65 \%$ of the maximum heart rate, the participants of the HIIT group conducted 5 high-intensity intervals of 1.5 minutes at $95 \%-100 \%$ of their maximum heart rate with 2 minutes of unloaded pedaling in between.

\section{Outcomes and Assessments}

To investigate acute (single-bout) and training (3-week intervention) effects, blood samples were collected immediately before $\left(t_{0}\right)$, immediately after $\left(t_{1}\right)$, and 3 hours after the first exercise session $\left(t_{2}\right)$ as well as after the training intervention $\left(t_{3}\right)$. At $t_{0}$ and $t_{3}$, blood samples were collected in a resting condition, without any amount of cardiorespiratory arousal due to exercise in the past 24 hours. Blood samples were taken between 8 and 9 AM. Participants were told to refrain from any nutritional intake before blood samples were collected.

After blood samples were drawn from the medial cubital vein in a supine position, samples were rested at room temperature for 10 minutes for clotting. Subsequently, samples were centrifuged at 3,500 rpm for 10 minutes. Plasma samples were aliquoted and stored at $-80^{\circ} \mathrm{C}$ until further analyses. All outcome assessors were blinded to clinical data and intervention group allocation. 
Table 1 Participant Baseline Characteristics

\begin{tabular}{|c|c|c|c|}
\hline & HIIT $(n=35)$ & MCT $(n=34)$ & Overall $(n=69)$ \\
\hline $\operatorname{Sex}(F / M)$ & $24 / 11$ & $19 / 15$ & $43 / 26$ \\
\hline Age (y) & $50.89(10.31)$ & $49.65(10.04)$ & $50.28(10.12)$ \\
\hline Height (cm) & $170.94(8.57)$ & $171.79(7.56)$ & $171.19(8.15)$ \\
\hline Weight (kg) & $73.85(14.60)$ & $74.90(16.30)$ & $74.37(15.36)$ \\
\hline BMI $\left(\mathbf{k g} \cdot \mathrm{m}^{-2}\right)$ & $25.28(4.09)$ & $25.26(4.76)$ & $25.27(4.40)$ \\
\hline EDSS score & $4.44(1.06)$ & $4.59(1.08)$ & $4.51(1.06)$ \\
\hline MS phenotype (RRMS/SPMS) & $21 / 14$ & $21 / 13$ & $42 / 27$ \\
\hline Time since diagnosis $(y)$ & $14.8(8.26)$ & $12.24(7.5)$ & $13.54(7.94)$ \\
\hline DMT (yes/no) (\%) & $78.8 / 24.2$ & $81.8 / 18.2$ & $79.1 / 20.9$ \\
\hline Years since last relapse $(y)$ & $5.32(4.59)$ & $4.53(3.03)$ & $4.95(3.92)$ \\
\hline $\mathrm{VO}_{\text {peak }}\left(\mathrm{mL} \cdot \mathrm{min}^{-1}\right)$ & $1,416.06(448.71)$ & $1,405.79(402.17)$ & $1,411.00(423.31)$ \\
\hline $\mathrm{VO}_{\text {peak }}\left(\mathrm{mL} \cdot \mathrm{kg}^{-1} \cdot \mathrm{min}^{-1}\right)$ & $19.30(5.64)$ & $19.09(5.13)$ & $19.20(5.36)$ \\
\hline Watt $_{\max }(\mathrm{W})$ & $98.91(39.82)$ & $96(32.23)$ & $97.48(36.04)$ \\
\hline Watt $_{\text {rel }}\left(\mathrm{W} \cdot \mathbf{k g}^{-1}\right)$ & $1.36(0.55)$ & $1.31(0.45)$ & $1.34(0.50)$ \\
\hline SDMT (points) & $44.14(10.30)$ & $42.88(14.07)$ & $43.53(12.20)$ \\
\hline VLMT (total score. points) & $52.43(8.74)$ & $51.73(11.71)$ & $52.09(10.22)$ \\
\hline BVMT-R (total score. points) & $21.03(7.33)$ & $19.55(7.67)$ & $20.31(7.48)$ \\
\hline
\end{tabular}

Abbreviations: BMI = body mass index; BVMT-R = Brief Visuospatial Memory Test-Revised; DMT = disease-modifying treatment; EDSS = Expanded Disability Status Scale; HIIT = high-intensity interval training; MCT = moderate continuous training; RRMS = relapsing-remitting MS; SDMT = Symbol Digit Modalities Test, SPMS = secondary progressive MS; VLMT = verbal learning memory test; $\mathrm{VO}_{2 \text { peak }}=$ peak oxygen uptake; Watt $_{\text {max }}=$ peak power output; Watt $_{\text {rel }}=$ relative (referring to bodyweight) peak power output. Values are presented as mean (SD).

\section{pNfL Assessment}

As described for serum measurements, ${ }^{15,16}$ the $\mathrm{pNfL}$ level was determined by means of a SiMoA HD-1 device (Quanterix, Billerica, MA) using NF-Light Advantage Kits (Quanterix) of a single LOT according to the manufacturer's instructions. Although both the samples and the remaining kit components were allowed to come to room temperature, resorufin- $\beta-D-$ galactopyranoside was incubated at $33^{\circ} \mathrm{C}$ for 60 minutes. Samples were then vortexed and centrifuged for 5 minutes at $10,000 \mathrm{~g}$. Finally, all samples were measured in duplicates, and the coefficient of variation ( $\mathrm{CV}$, as a percentage) was obtained by dividing the SD by the mean value of both replicates multiplied by 100 . Samples with a sample CV above $20 \%$ (or missing replicate result) were measured a second time. The mean intra-assay $\mathrm{CV}$ was $7.3 \%$. Run-to-run variation was monitored by adding duplicates of the same 2 low and high controls, consisting of recombinant human NfL antigen, to each sample run. The mean levels over all runs were $3.0 \mathrm{pg} /$ $\mathrm{mL}$ for the low control and $137.9 \mathrm{pg} / \mathrm{mL}$ for the high control with interassay CVs of $4.4 \%$ and $1.5 \%$, respectively.

\section{Assessment of KYN Pathway Metabolites}

Plasma concentration of TRP, KYN, kynurenic acid (KA), and QA was assessed using high-performance liquid chromatography-tandem mass spectrometry (HPLC-MS/
MS) according to previously published protocols ${ }^{17,18}$ after precipitation of plasma proteins. To provide information on the KYN pathway regulation, the ratios of KYN/TRP, KA/ $\mathrm{KYN}$, QA/KYN, and QA/KA were calculated and defined as outcomes. Detailed information on HPLC-MS/MS is provided in supplement 1 (links.lww.com/NXI/A455).

\section{Assessment of Interleukin- 6}

Plasma levels of interleukin-6 (IL-6) were assessed using ELISA (Human IL-6 Quantikine HS ELISA Kit, R\&D Systems). Analysis was conducted according to the manufacturer's instructions. Levels of IL-6 were additionally assessed to consider an inflammatory marker that is relevant as KYN pathway mediator and myokine, which is known to be influenced by physical exercise.

\section{Statistical Analyses}

Correlation coefficients were calculated to examine potential baseline associations between $\mathrm{pNfL}$ and cognitive performance as well as between $\mathrm{IL}-6$, pNfL, and KYN pathway outcomes. To evaluate acute within- and between-group effects, baselineand IL-6-adjusted analysis of covariance models with repeated measures was performed. Sphericity was checked and adjusted using Greenhouse-Geisser correction if necessary. In case of significant main effects, Bonferroni-corrected post hoc comparisons were applied. Based on the observed acute 
Figure 1 Baseline Correlations (Spearman Coefficient) Between Cognitive Performance and pNfL Levels
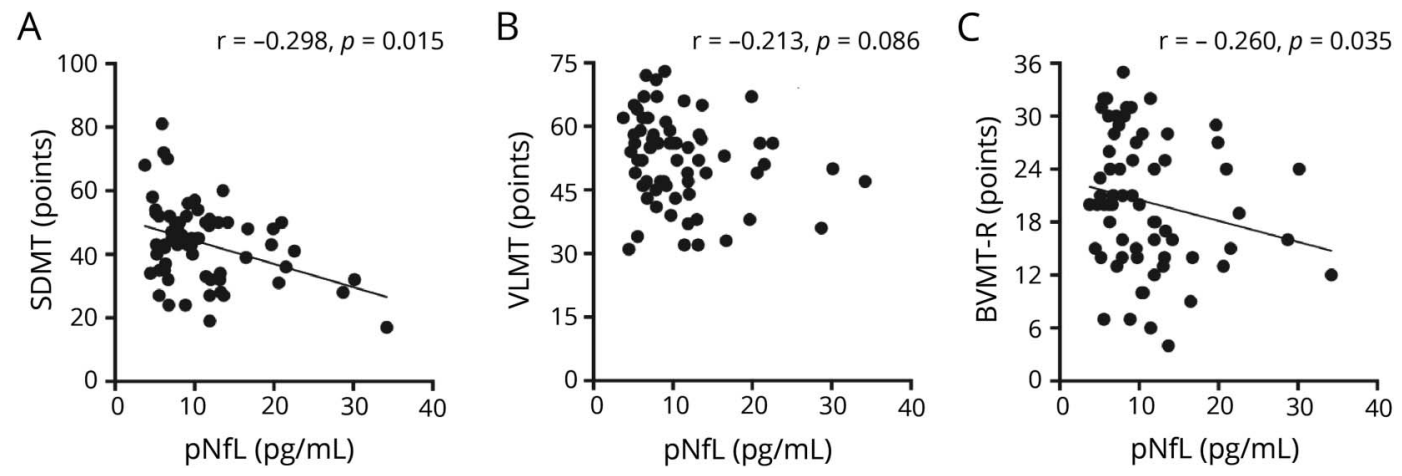

(A) Correlation between processing speed and pNfL levels. (B) Correlation between verbal learning and pNfL levels. (C) visuospatial memory and pNfL levels. BVMT-R = Brief Visuospatial Memory Test-Revised; pNfL = plasma neurofilament light chain; SDMT = Symbol Digit Modalities Test; VLMT = Verbal Learning and Memory Test.

effects on pNfL and the neuroprotective KYN pathway branch toward KA, we performed delta correlations subsequently. To evaluate training effects, postintervention values were compared between groups in univariate baseline- and IL- 6 adjusted analysis of covariance models. Outliers defined as $>3$ SD were excluded before analyses. Level of significance was set at $p \leq 0.05$. Statistical analyses were conducted using SPSS 26 (IBM, Armonk, NY).

\section{Data Availability}

Data from this study are available from the authors on reasonable request.

\section{Results}

\section{Baseline Associations Between pNfL Levels, Cognitive Performance, and KYN Pathway Outcomes}

Baseline correlations indicated negative associations between pNfL and processing speed (Symbol Digit Modalities Test) $(r=-0.298 ; p=0.015)$ as well as visuospatial memory (Brief Visuospatial Memory Test-Revised) $(r=-0.260, p=0.035)$, whereas the association between pNfL and verbal learning (Verbal Learning and Memory Test) did not reach statistical significance (figure 1).

Table 2 Baseline Correlations Between IL-6, pNfL, and KYN Pathway Outcomes

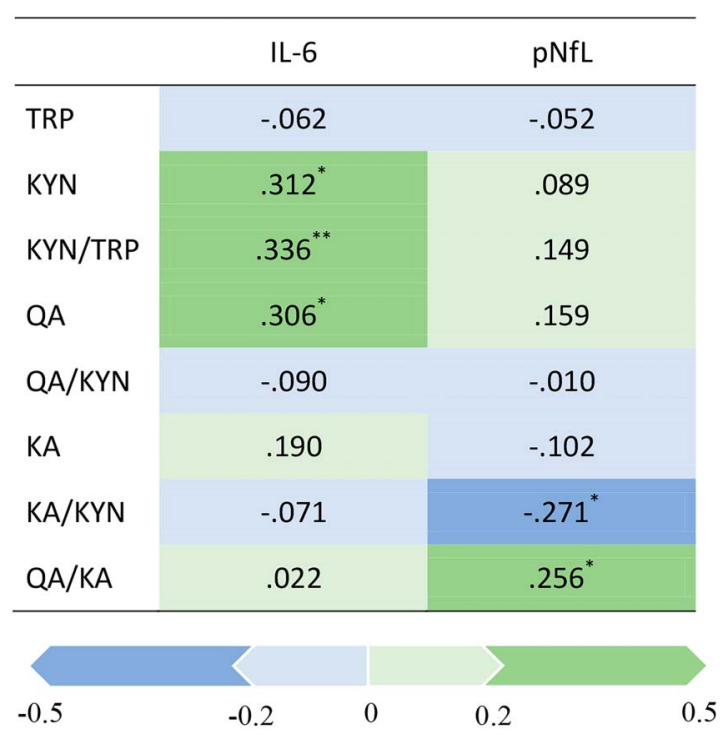

Graphic representation of Spearman's correlation coefficient.

Abbreviations: IL-6 = interleukin-6; KA = kynurenic acid; KA/KYN = kynurenic acid-to-kynurenine ratio; KYN = kynurenine; KYN/TRP = kynurenine-to-tryptophan ratio; pNfL = plasma neurofilament light chain; $\mathrm{QA}=$ quinolinic acid; QA/KA = quinolinic acid-to-kynurenic acid ratio; $\mathrm{QA} / \mathrm{KYN}$ = quinolinic acid-to-kynurenine ratio; TRP = tryptophan.

${ }^{*} p \leq 0.05 ;{ }^{*} p \leq 0.01$. 
Figure 2 Acute Kinetics of pNfL (A), IL-6 (B), and KYN Pathway Outcomes (C-J) Between High-Intensity Interval Training vs Moderate Continuous Training
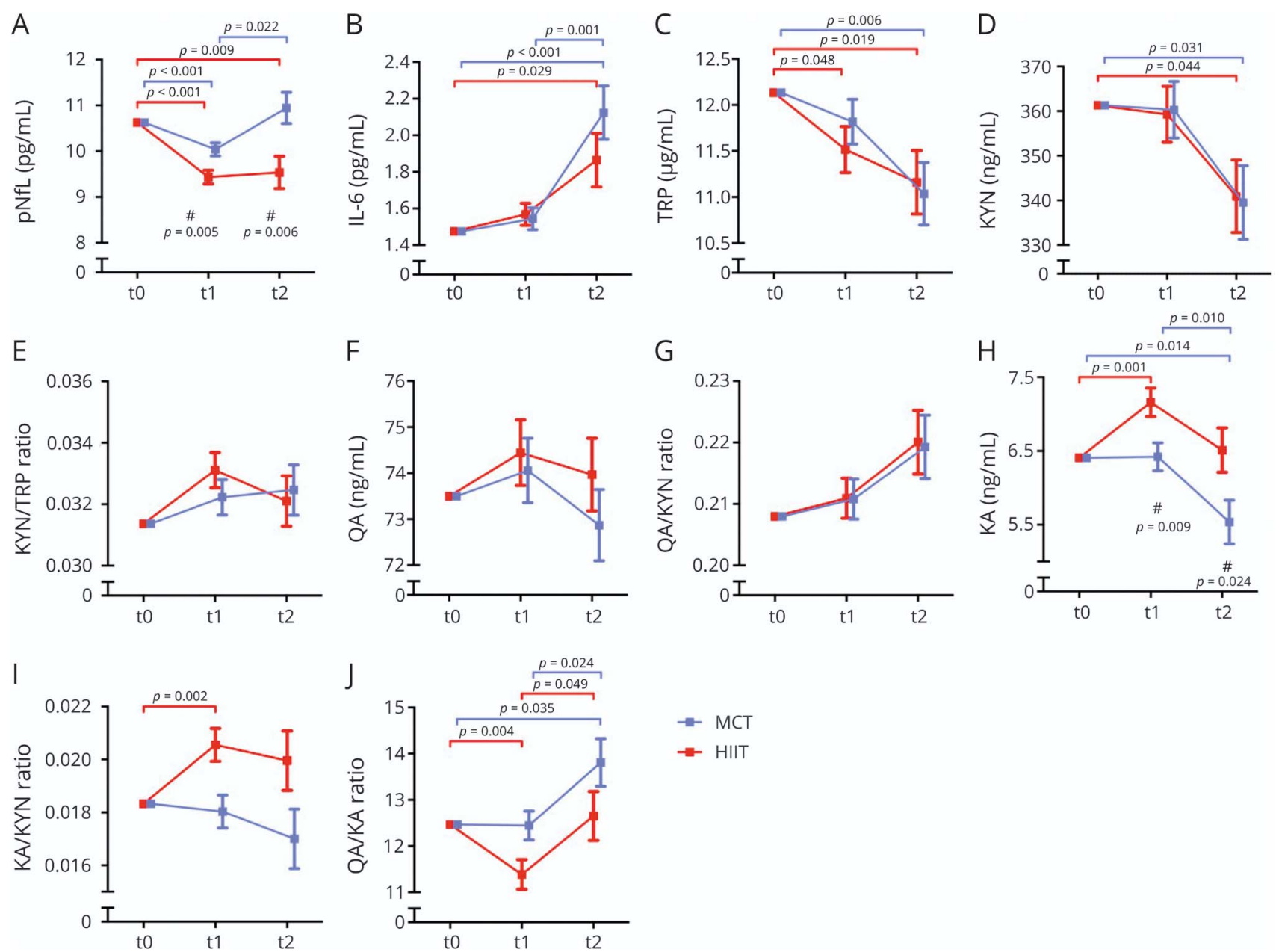

Data shown as baseline- and IL-6-adjusted outcome kinetics (mean \pm SEM) separated by intervention groups. \#Significant between-group effect. IL-6 = interleukin-6; KA = kynurenic acid; KA/KYN = kynurenic acid-to-kynurenine ratio; $\mathrm{KYN}=$ kynurenine; $\mathrm{KYN} / \mathrm{TRP}=$ kynurenine-to-tryptophan ratio; pNfL = plasma neurofilament light chain; $\mathrm{QA}=$ quinolinic acid; $\mathrm{QA} / \mathrm{KA}=$ quinolinic acid-to-kynurenic acid ratio; $\mathrm{QA} / \mathrm{KYN}$ = quinolinic acid-to-kynurenine ratio; $\mathrm{t}_{0}=$ baseline; $\mathrm{t}_{1}=$ immediately postexercise; $t_{2}=3$ hours postexercise; TRP = tryptophan.

Levels of IL-6 correlated positively with KYN $(r=0.312 ; p=$ $0.011), \mathrm{KYN} / \mathrm{TRP}$ ratio $(r=0.306 ; p=0.013)$, and QA $(r=$ $0.336 ; p=0.006)$. A negative correlation between $\mathrm{pNfL}$ and the KA/KYN ratio $(r=-0.271 ; p=0.029)$ was observed, whereas pNfL correlated positively with the QA/KA ratio $(r=$ 0.256; $p=0.040$ ). Baseline correlations between IL-6, pNfL, and KYN pathway outcomes are illustrated in table 2.

\section{Acute Effects of HIIT Versus MCT on pNfL and KYN Pathway Outcomes}

Levels of pNfL decreased within MCT from baseline to immediately postexercise $(p<0.001$; CI $[-0.940$ to -0.237$])$ and subsequently increased to 3 hours postexercise $(p=$ 0.022; CI [0.104-1.711]). pNfL decreased from baseline to immediately postexercise $(p<0.001$; CI $[-1.555$ to -0.830$])$ and 3 hours postexercise ( $p=0.009$; CI $[-1,961$ to -0.219$])$ within HIIT. A significant interaction effect between the groups was observed at both measurement time points, immediately postexercise $(p=0.005$; CI $[-1.015$ to -0.193$)$ and 3 hours postexercise $(p=0.006$; CI $[-2.396$ to -0.422$])$, with lower pNfL levels in HIIT compared with MCT.

The acute bout of exercise led to an increase in IL- 6 within MCT from baseline to 3 hours postexercise ( $p<0.001 ; 95 \%$ CI $[0.290-1.008])$ and from immediately postexercise to 3 hours postexercise $(p=0.001$; CI $[0.197-0.964])$. Within HIIT, IL-6 increased from baseline to 3 hours postexercise ( $p$ $=0.029 ; \mathrm{CI}[0.030-0.748])$. No interaction effect between groups was observed.

Levels of TRP decreased within MCT from baseline to 3 hours postexercise ( $p=0.006$; CI $[-1.933$ to -00.262$])$. Within HIIT, TRP decreased from baseline to immediately postexercise $(p=0.048$; CI $[-1.230$ to -0.004$])$ as well as to 3 hours postexercise $(p=0.019$; CI $[-1.822$ to -0.126$])$. No interaction effects were observed for TRP. Levels of KYN 
Table 3 Delta Correlations Between Acute Changes in pNfL ( $\left.t_{2}-t_{0}\right)$ and in KYN Pathway Outcomes Toward Kynurenic Acid

\begin{tabular}{|c|c|c|c|c|c|c|}
\hline & $\begin{array}{c}\Delta \mathrm{KA} \\
\left(\mathrm{t}_{1}-\mathrm{t}_{0}\right)\end{array}$ & $\begin{array}{r}\Delta \mathrm{KA} \\
\left(\mathrm{t}_{2}-\mathrm{t}_{0}\right)\end{array}$ & $\begin{array}{c}\Delta \mathrm{KA} / \mathrm{KYN} \\
\left(\mathrm{t}_{1}-\mathrm{t}_{0}\right)\end{array}$ & $\begin{array}{c}\Delta \mathrm{KA} / \mathrm{KYN} \\
\left(\mathrm{t}_{2}-\mathrm{t}_{0}\right)\end{array}$ & $\begin{array}{c}\Delta Q A / K A \\
\left(t_{1}-t_{0}\right)\end{array}$ & $\begin{array}{c}\triangle Q A / K A \\
\left(t_{2}-t_{0}\right)\end{array}$ \\
\hline $\begin{array}{c}\Delta p N f L \\
\left(t_{2}-t_{0}\right)\end{array}$ & $-.254^{*}$ & -.221 & $-.264^{*}$ & -.118 & $.412^{* *}$ & $.304^{*}$ \\
\hline & & & $<$ & $\sum$ & & \\
\hline & & & -0.2 & 0.2 & 0.5 & \\
\hline
\end{tabular}

Graphic representation of Spearman's correlation coefficient.

Abbreviations: $\Delta$ = delta; IL-6 = interleukin-6; KA = kynurenic acid; KA/KYN = kynurenic acid-to-kynurenine ratio; pNfL = plasma neurofilament light chain; QA $\mathrm{KA}=$ quinolinic acid-to-kynurenic acid ratio; $\mathrm{t}_{0}=$ baseline; $\mathrm{t}_{1}=$ immediately postexercise; $\mathrm{t}_{2}=3$ hours postexercise.

${ }^{*} p \leq 0.05 ;{ }^{* *} p \leq 0.01$.

decreased from baseline to 3 hours postexercise within $\operatorname{MCT}(p=0.031 ; \mathrm{CI}[-42.133$ to -1.483$])$ as well as within HIIT ( $p=0.044$; CI $[-40.425$ to -0.401$])$, but no effects between the groups were observed. Regarding KYN/TRP ratio, QA, and QA/KYN ratio, neither significant withinnor between-group effects were observed. Levels of KA decreased within MCT from baseline to 3 hours postexercise $(p=0.014$; CI $[-1.600$ to -0.143$])$ as well as from immediately postexercise to 3 hours postexercise ( $p=$ 0.010; CI [-1.603 to -0.171$])$. Within HIIT, KA increased from baseline to immediately postexercise $(p=0.001$; CI [0.273-1.230]). Between-group differences were observed immediately postexercise $(p=0.009$; CI $[-0.1 .281$ to $-0.190])$ and 3 hours hours postexercise $(p=0.024$; CI $[-1.820$ to -0.133$)$, with greater KA levels in HIIT than in MCT. The KA/KYN ratio increased within HIIT from baseline to immediately postexercise $(p=0.002$; CI [0.001-0.004]), whereas no other significant effects within or between the groups were detected. The QA/KA ratio increased significantly in MCT from baseline to 3 hours postexercise $(p=0.035$; CI $[0.074-2.613])$ as well as from postexercise to 3 hours postexercise $(p=0.024$; CI [0.137-2.590]). Within HIIT, the QA/KA ratio decreased from baseline to immediately postexercise ( $p=0.004$; CI $[-1.881$ to -0.285$])$ and subsequently increased to 3 hours postexercise $(p=0.049$; CI $[0.002-2.535])$. No betweengroup effects for the QA/KA ratio were observed. Acute kinetics of HIIT vs MCT on IL-6, pNfL, and KYN pathway outcomes are shown in figure 2 .

\section{Correlations of Acute Changes in $\mathrm{PNfL}$ and the KYN Pathway Toward KA}

Delta correlations (table 3) of acute exercise-induced alterations indicated a negative association between the changes in $\mathrm{KA}(r=-0.254 ; p=0.043)$ and the $\mathrm{KA} / \mathrm{KYN}$ ratio $(r=$ $-0.264 ; p=0.037)$ from baseline to immediately postexercise and changes in pNfL from baseline to 3 hours postexercise. Moreover, positive correlations between the change in $\mathrm{pNfL}$ from baseline to 3 hours postexercise and changes in the QA/
KA ratio $\left(\Delta \mathrm{t}_{1}-\mathrm{t}_{0} r=0.412 ; p=0.001\right.$ and $\Delta \mathrm{t}_{2}-\mathrm{t}_{0} r=0.304 ; p=$ $0.016)$ were revealed.

\section{Training Effects on pNfL Levels and KYN Pathway Outcomes}

Concerning training effects, a significant difference between HIIT and MCT was observed following the 3-week intervention period (figure 3 ). The KYN/TRP ratio was significantly greater in HIIT compared with MCT ( $p=$ $0.032)$. No significant training effects were observed for pNfL, IL-6, TRP, KYN, QA, KA, QA/KYN ratio, KA/KYN ratio, and $\mathrm{QA} / \mathrm{KA}$ ratio. Detailed analysis of covariance results for both acute and training effects are reported in supplement 2 (links.lww.com/NXI/A456). Raw data shown as mean \pm SD are provided in supplement 3 (links. lww.com/NXI/A457).

\section{Classification of Evidence}

This secondary investigation was designed to examine acute and training effects of HIIT as the intervention of interest compared with standard exercise therapy on pNfL levels and KYN pathway metabolites in moderately disabled pwMS. In addition, we exploratory investigated a potential association between changes in pNfL levels and changes in the KYN pathway flux toward the neuroprotective KA.

This randomized controlled clinical interventional trial provides class II evidence that acute HIIT diminishes $\mathrm{pNfL}$ and increases the metabolic flux of the KYN pathway toward KA. Moreover, this study provides Class II evidence that a 3-week HIIT intervention increases the KYN/TRP ratio as marker of KYN pathway activation compared with standard exercise therapy in pwMS.

\section{Discussion}

Here, we showed that acute exercise reduces pNfL levels in pwMS in an intensity-dependent manner. This pNfL reduction is associated with $\mathrm{KYN}$ pathway rerouting toward the neuroprotective and immunosuppressive metabolite KA. 
Figure 3 Training Effects of High-Intensity Interval Training vs Moderate Continuous Training on pNfL (A), IL-6 (B), and KYN Pathway Outcomes (C-J)

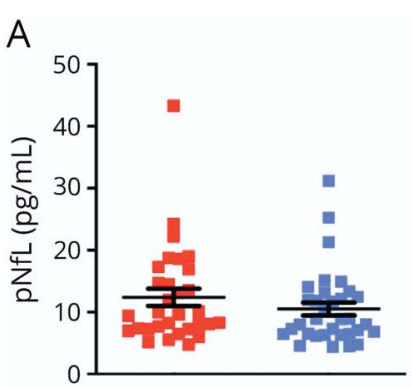

E

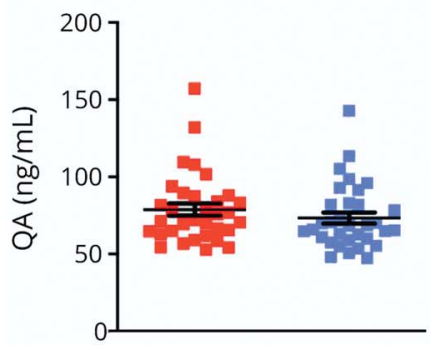

I

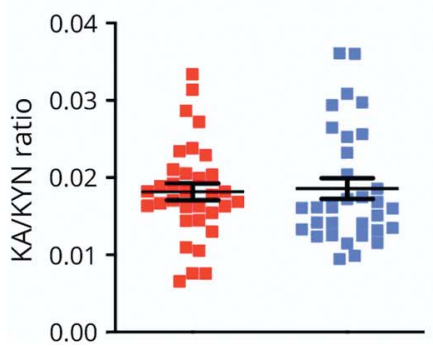

B

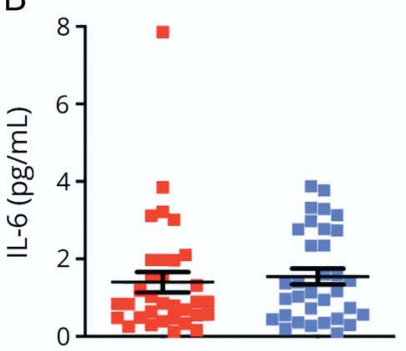

$\mathrm{F}$

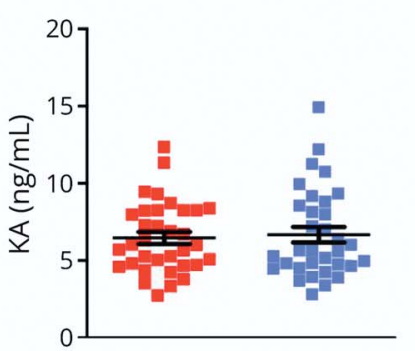

J

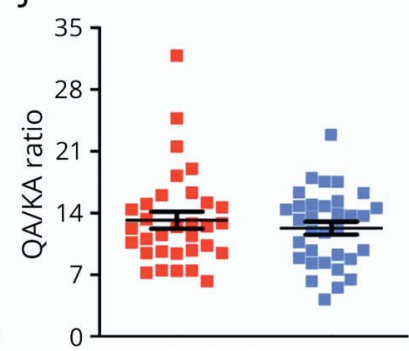

C

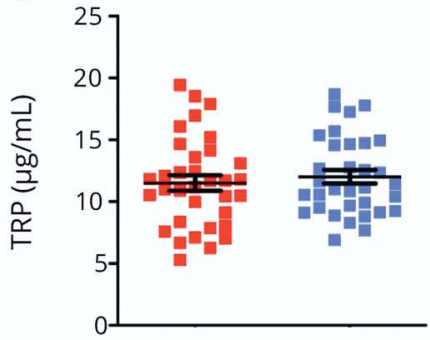

G
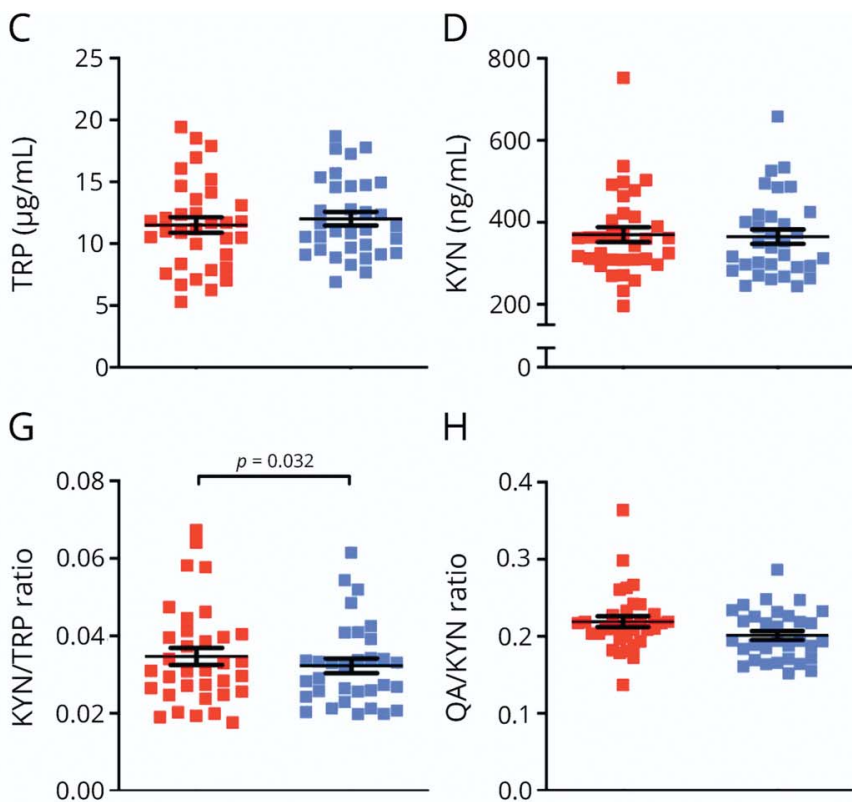

$\mathrm{H}$

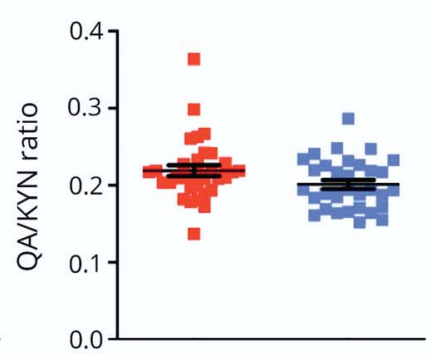

Data shown as postexercise intervention values $\left(t_{3}\right)$ separated by intervention groups. Illustrated significance is based on baseline- and IL-6-adjusted ANCOVA results. ANCOVA = analysis of covariance; IL- 6 = interleukin-6; KA = kynurenic acid; KA/KYN = kynurenic acid-to-kynurenine ratio; $\mathrm{KYN}=$ kynurenine; $\mathrm{KYN} / \mathrm{TRP}=$ kynurenine-to-tryptophan ratio; $\mathrm{pNfL}=$ plasma neurofilament light chain; $\mathrm{QA}$ = quinolinic acid; QA/KA = quinolinic acid-to-kynurenic acid ratio; QA/ $\mathrm{KYN}=$ quinolinic acid-to-kynurenine ratio; TRP = tryptophan.

Baseline pNfL levels correlated negatively with participants' cognitive performance, underlining the integrative nature and clinical relevance of $\mathrm{pNfL}$ as peripheral blood biomarker in pwMS. Baseline pNfL levels were also associated with KYN pathway parameters. Although the $\mathrm{KA} / \mathrm{KYN}$ ratio correlated negatively, the QA/KA ratio correlated positively with $\mathrm{pNfL}$ levels. This observation fits into the literature that attributes KA as neuroprotective and QA as neurotoxic agent of the KYN pathway, respectively. ${ }^{19}$ Furthermore, we showed consistent baseline correlations between IL-6 levels and KYN pathway activation as well as between IL-6 and QA. These associations indicate a link between the inflammatory state and KYN pathway activation toward QA within this sample of pwMS.

The results show that both acute HIIT and MCT reduce pNfL levels, but HIIT induces a greater reduction compared with MCT. Levels of pNfL were still decreased 3 hours after exercise completion in HIIT suggesting a more sustainable effect. Further mechanistic investigations focusing on the neuroprotective effects of single exercise bouts as reflected by the decrease in pNfL following acute exercise in pwMS are highly warranted to improve our knowledge about how exercise-induced benefits on symptoms and clinical outcomes in neurodegenerative diseases are achieved. Moreover, future clinical trials as well as routine assessments of $\mathrm{pNfL}$ should consider acute physical exercise as confounding factor for measurement reliability.

Regarding acute effects on the KYN pathway, we observed a catabolic shift in favor of the end product KA that is in line with previous investigations in other populations. ${ }^{17}$ Greater effects of acute exercise on KA levels were observed in HIIT compared with MCT at both postexercise measurement time points. This finding confirms the results of previous studies in both animals and humans showing an elevated KAT expression in skeletal muscle and immune cells. ${ }^{17,20}$ An increase in $\mathrm{KA}$ is possibly not only important for neuroprotective properties within the CNS but also concerning its immunomodulatory effects. KA represents a well-described endogenous aryl hydrocarbon receptor (AhR) ligand, which mediates the 


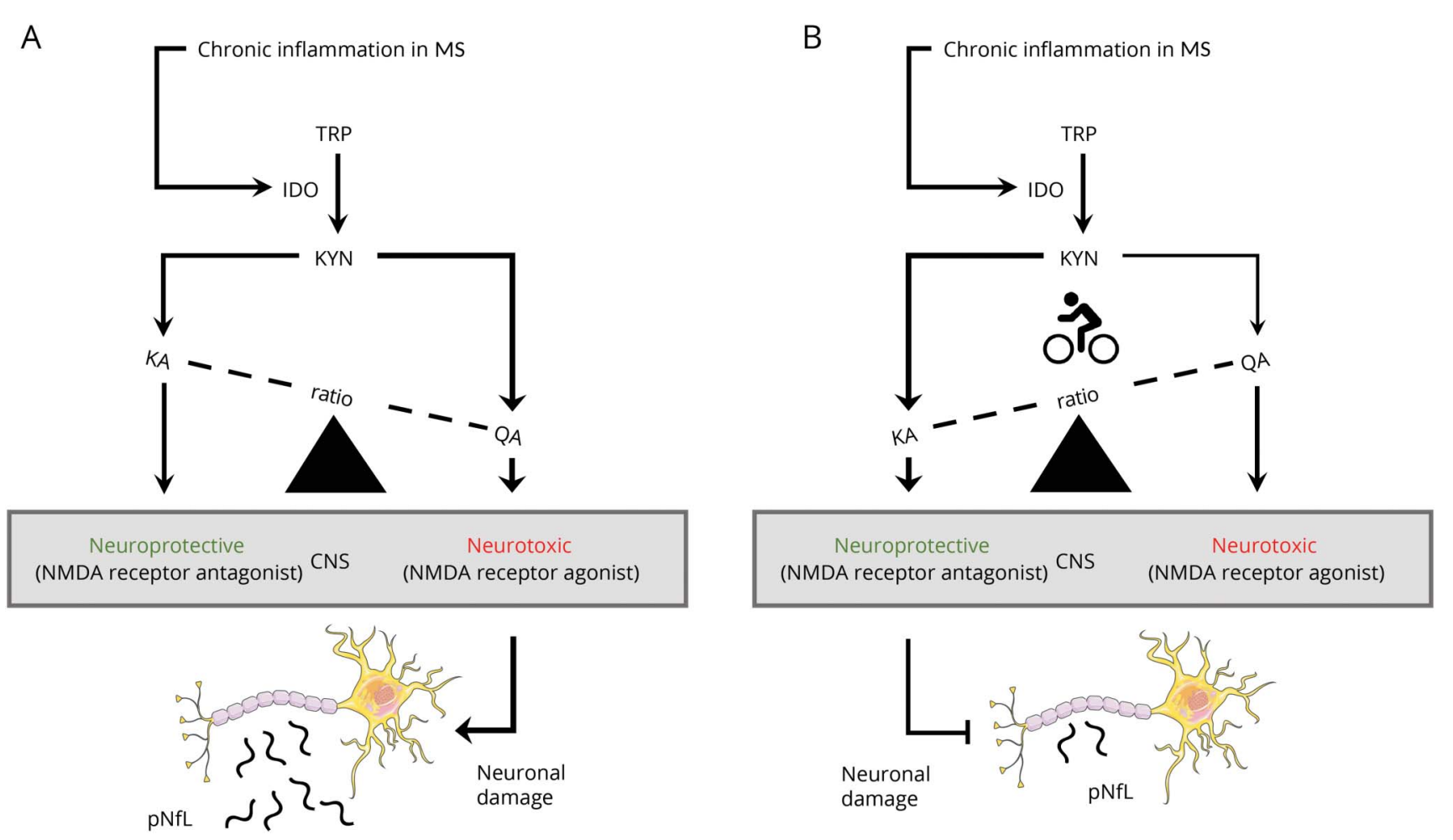

(A) Interaction of chronic inflammatory conditions in MS, the KYN pathway, and neurodegeneration assessed by pNfL levels. (B) Exercise-induced rerouting of the $\mathrm{KYN}$ pathway toward $\mathrm{KA}$ leads to reductions in $\mathrm{pNfL}$ levels. $\mathrm{KA}=$ kynurenic acid; $\mathrm{KYN}=$ kynurenine; $\mathrm{pNfL}=$ plasma neurofilament light chain; $\mathrm{QA}=$ quinolinic acid; TRP = tryptophan.

differentiation of naive $\mathrm{CD} 4^{+} \mathrm{T}$-helper cells to immunosuppressive regulatory $\mathrm{T}$ cells following ligand-dependent activation. $^{21}$ This increased AhR ligand availability in blood plasma provides the foundation for upcoming trials.

Furthermore, the acute exercise-induced changes in KA and in pNfL were associated negatively. We also observed a positive association between changes in $\mathrm{pNfL}$ and in the $\mathrm{QA} / \mathrm{KA}$ ratio. These findings suggest the hypothesis that the short-term increase in KA and consequently the shift of the QA/KA balance leads to neuroprotection as reflected by the reduction in pNfL levels (figure 4).

In contrast to profound acute effects on pNfL and the KYN pathway flux toward KA, solely one significant alteration following the training period was observed. Compared with MCT, an elevated KYN/TRP ratio as parameter of initial KYN pathway activation was detected in HIIT that is in line with results from a previous study. ${ }^{22} \mathrm{~A}$ recent EAE mouse model revealed that peripheral IDO boosting for the purpose of KYN pathway activation provokes dendritic cells within lymphatic tissue to suppress the generation of myelin oligodendrocyte glycoprotein (MOG) $\mathrm{T}$ cells, which induce neuronal damage. ${ }^{23}$ Aside from MOG $\mathrm{T}$ cells, evidence indicates that increased IDO activity enhances the differentiation of regulatory $\mathrm{T}$ cells, ${ }^{24}$ suppressing immune overactivation in MS. Although only an elevated KYN/
TRP ratio was observed following the HIIT intervention, this finding further suggests disease-modifying properties of exercise in pwMS and encourages future research to uncover the mentioned neuroimmunologic consequences. However, considering the acute decreases in pNfL levels after HIIT, a reduction following the training intervention remains absent, which is in line with studies investigating the effect of exercise or multimodal rehabilitation on serum NfL levels in other neurologic disorders. $^{25,26}$ Further mechanistic research focusing on the interaction between acute and longer-term training effects on $\mathrm{pNfL}$ is needed to understand why pwMS benefit from physical exercise and whether chronic exercise training leads to an alleviation in disease progression.

Although the observed persistent acute decrease in pNfL suggests a chronic reduction following repetitive acute exercise bouts, no training effects on pNfL levels were detected. Nevertheless, 2 major methodological aspects need to be considered. First, the 3-week intervention is very short to assess training effects. A longer intervention period is possibly necessary to reach a chronic reduction in pNfL. However, inpatient rehabilitation usually does not last longer than 4 weeks for pwMS, leading to the question whether this time span is sufficient for conspicuous improvements. Second, we did not include a passive control group due to ethical reasons. In addition, regarding acute effects, we did not compare the 
results with a passive resting group, yet it needs to be taken into account that the present results even show superior effects of HIIT compared with the standard exercise therapy. Both groups exercised $3 \times /$ week, and only the exercise modalities differed. The importance of higher intensities is underlined by the reported acute effects and supports findings of earlier investigations. ${ }^{11,12}$

The investigation of the KYN metabolites in peripheral blood provides knowledge about the systemic balance of the KYN pathway and the availability of neuroactive metabolites for the CNS. Nonetheless, the conclusions drawn on neuromodulatory properties remain speculative because $\mathrm{KYN}$ pathway metabolites were not assessed in the CSF. However, consistent correlations between CSF and plasma/serum levels of KYN pathway metabolites have been reported, ${ }^{6}$ and, even more importantly, we revealed correlations between the neuroprotective KYN pathway branch toward $\mathrm{KA}$ and pNfL levels as marker of neuronal damage.

Acute exercise diminishes pNfL levels in pwMS, indicating a potential alleviation in ongoing neurodegeneration. Clinicians and researchers should consider acute physical exercise as confounding factor for the assessment of pNfL levels. Exercisemediated KYN pathway rerouting toward KA as end product is associated with pNfL reductions and might be responsible for neuroprotective effects. Overall, HIIT leads to greater responses than MCT, emphasizing the importance of higher exercise intensities when exercising with pwMS. Future studies are needed to profoundly address this promising mechanistic link between neuroprotective properties, neuroinflammation, and exercise in pwMS and other neurodegenerative disorders. Finally, the consequences of repetitively occurring exerciseinduced acute effects on pNfL levels and KYN pathway regulation require further research approaches that consider additional (intracellular) biomarkers of physical stress. The KYN/TRP upregulation following 3 weeks of HIIT suggests disease-counterregulatory properties of exercise on immune homeostasis, which remains to be investigated.

\section{Acknowledgment}

The authors thank Daniel Caminada and Francesca Ferrara from Labor Risch for support with blood sample preparation and storage. Furthermore, the authors thank Ramona Sylvester for supervising exercise training sessions.

\section{Study Funding}

German MS Society; Swiss MS Society (SMSG-12D2018); Grenzen überschreiten (GRENZ-JB/18-007); Blumenau-Léonie Hartmann-Stiftung; Stiftung Ergotherapie (ergo-ZH-28-2018).

\section{Disclosure}

The authors declare that they have no conflict of interest. C. Warnke: institutional support: Biogen, Novartis, Roche, Sanofi, and Alexion. Go to Neurology.org/NN for full disclosures.

\section{Publication History}

Received by Neurology: Neuroimmunology \& Neuroinflammation October 21, 2020. Accepted in final form January 21, 2021.

Appendix Authors

\begin{tabular}{|c|c|c|}
\hline Name & Location & Contribution \\
\hline $\begin{array}{l}\text { Niklas } \\
\text { Joisten, PhD }\end{array}$ & $\begin{array}{l}\text { Department of } \\
\text { "Performance and Health } \\
\text { (Sports Medicine)", } \\
\text { Institute of Sport and } \\
\text { Sport Science, Technical } \\
\text { University Dortmund, } \\
\text { Germany }\end{array}$ & $\begin{array}{l}\text { Drafting/revision of the } \\
\text { manuscript for content, } \\
\text { including medical } \\
\text { writing for content; } \\
\text { major role in the } \\
\text { acquisition of data; and } \\
\text { analysis or } \\
\text { interpretation of data }\end{array}$ \\
\hline $\begin{array}{l}\text { Annette } \\
\text { Rademacher }\end{array}$ & $\begin{array}{l}\text { Department for Molecular } \\
\text { and Cellular Sports } \\
\text { Medicine, Institute of } \\
\text { Cardiovascular Research } \\
\text { and Sports Medicine, } \\
\text { German Sport University } \\
\text { Cologne }\end{array}$ & $\begin{array}{l}\text { Drafting/revision of the } \\
\text { manuscript for content, } \\
\text { including medical writing } \\
\text { for content; major role in } \\
\text { the acquisition of data; } \\
\text { and analysis or } \\
\text { interpretation of data }\end{array}$ \\
\hline $\begin{array}{l}\text { Clemens } \\
\text { Warnke, MD }\end{array}$ & $\begin{array}{l}\text { Department of } \\
\text { Neurology, Faculty of } \\
\text { Medicine and University } \\
\text { Hospital Cologne, } \\
\text { University of Cologne, } \\
\text { Germany }\end{array}$ & $\begin{array}{l}\text { Drafting/revision of the } \\
\text { manuscript for content, } \\
\text { including medical writing } \\
\text { for content, and study } \\
\text { concept or design }\end{array}$ \\
\hline $\begin{array}{l}\text { Sebastian } \\
\text { Proschinger }\end{array}$ & $\begin{array}{l}\text { Department for } \\
\text { Molecular and Cellular } \\
\text { Sports Medicine, Institute } \\
\text { of Cardiovascular } \\
\text { Research and Sports } \\
\text { Medicine, German Sport } \\
\text { University Cologne, } \\
\text { Germany }\end{array}$ & $\begin{array}{l}\text { Drafting/revision of the } \\
\text { manuscript for content, } \\
\text { including medical writing } \\
\text { for content, and major role } \\
\text { in the acquisition of data }\end{array}$ \\
\hline
\end{tabular}

\begin{tabular}{lll}
\hline $\begin{array}{l}\text { Alexander } \\
\text { Schenk, PhD }\end{array}$ & $\begin{array}{l}\text { Department of } \\
\text { "Performance and Health } \\
\text { (Sports Medicine), } \\
\text { Institute of Sport and } \\
\text { Sport Science, Technical } \\
\text { University Dortmund, } \\
\text { Germany }\end{array}$ & $\begin{array}{l}\text { Drafting/revision of the } \\
\text { manuscript for content, } \\
\text { including medical writing } \\
\text { for content, and major role } \\
\text { in the acquisition of data }\end{array}$ \\
\hline David Walzik & $\begin{array}{l}\text { Department of } \\
\text { "Performance and } \\
\text { Health (Sports Medicine)", } \\
\text { Institute of Sport } \\
\text { and Sport Science, } \\
\text { Technical University } \\
\text { Dortmund }\end{array}$ & $\begin{array}{l}\text { Drafting/revision of the } \\
\text { manuscript for content, } \\
\text { including medical writing } \\
\text { for content, and major role } \\
\text { in the acquisition of data }\end{array}$ \\
\hline
\end{tabular}

\begin{tabular}{lll}
\hline $\begin{array}{l}\text { Andre Knoop, } \\
\text { PhD }\end{array}$ & $\begin{array}{l}\text { Center for Preventive } \\
\text { Doping Research/Institute } \\
\text { of Biochemistry, German } \\
\text { Sport University Cologne, } \\
\text { Germany }\end{array}$ & $\begin{array}{l}\text { Analysis or interpretation } \\
\text { of data; additional } \\
\text { contributions: designed } \\
\text { and conducted outcome } \\
\text { measurement }\end{array}$ \\
\hline $\begin{array}{l}\text { Mario Thevis, } \\
\text { PhD }\end{array}$ & $\begin{array}{l}\text { Center for Preventive } \\
\text { Doping Research/ } \\
\text { Institute of Biochemistry, } \\
\text { German Sport University } \\
\text { Cologne }\end{array}$ & $\begin{array}{l}\text { Analysis or interpretation } \\
\text { of data; additional } \\
\text { contributions: designed } \\
\text { and conducted outcome } \\
\text { measurement }\end{array}$ \\
\hline Falk Steffen & $\begin{array}{l}\text { Department of Neurology, } \\
\text { Focus Program }\end{array}$ & $\begin{array}{l}\text { Analysis or interpretation } \\
\text { of data; additional } \\
\text { contributions: designed } \\
\text { and conducted outcome } \\
\text { meanslational } \\
\text { Neuroscience (FTN), and } \\
\text { Immunotherapy (FZl), } \\
\text { University Medical Center } \\
\text { of the Johannes } \\
\text { Gutenberg University, } \\
\text { Germany }\end{array}$ \\
& & \\
& & \\
& &
\end{tabular}


Appendix (continued)

\begin{tabular}{|c|c|c|}
\hline Name & Location & Contribution \\
\hline $\begin{array}{l}\text { Stefan } \\
\text { Bittner, MD }\end{array}$ & $\begin{array}{l}\text { Department of Neurology, } \\
\text { Focus Program } \\
\text { Translational } \\
\text { Neuroscience (FTN), and } \\
\text { Immunotherapy (FZI), } \\
\text { University Medical Center } \\
\text { of the Johannes } \\
\text { Gutenberg University, } \\
\text { Germany }\end{array}$ & $\begin{array}{l}\text { Analysis or interpretation } \\
\text { of data; additional } \\
\text { contributions: designed } \\
\text { and conducted outcome } \\
\text { measurement }\end{array}$ \\
\hline
\end{tabular}

\begin{tabular}{ll}
\hline Roman & Department of Neurology, Study concept or design \\
Gonzenbach, & Clinics of Valens, \\
MD & Rehabilitation Centre \\
& Valens, Switzerland
\end{tabular}

\begin{tabular}{|c|c|c|}
\hline Jan Kool, PhD & $\begin{array}{l}\text { Department of Neurology, } \\
\text { Clinics of Valens, } \\
\text { Rehabilitation Centre } \\
\text { Valens, Switzerland }\end{array}$ & Study concept or design \\
\hline $\begin{array}{l}\text { Wilhelm } \\
\text { Bloch, MD }\end{array}$ & $\begin{array}{l}\text { Department for Molecular } \\
\text { and Cellular Sports } \\
\text { Medicine, Institute of } \\
\text { Cardiovascular Research } \\
\text { and Sports Medicine, } \\
\text { German Sport University } \\
\text { Cologne, Germany }\end{array}$ & Study concept or design \\
\hline
\end{tabular}

PhD Clinics of Valens,
Rehabilitation Centre Valens, Switzerland

\section{Major role in the acquisition of data; study concept or design; and additional contributions: supervised manuscript drafting and obtained funding}

\begin{tabular}{|c|c|c|}
\hline $\begin{array}{l}\text { Philipp } \\
\text { Zimmer, PhD, } \\
\text { PhD }\end{array}$ & $\begin{array}{l}\text { Department of } \\
\text { "Performance and Health } \\
\text { (Sports Medicine)", } \\
\text { Institute of Sport and } \\
\text { Sport Science, Technical } \\
\text { University Dortmund, } \\
\text { Germany }\end{array}$ & $\begin{array}{l}\text { Study concept or design; } \\
\text { analysis or interpretation } \\
\text { of data; and additional } \\
\text { contributions: supervised } \\
\text { manuscript drafting and } \\
\text { obtained funding }\end{array}$ \\
\hline
\end{tabular}

\section{References}

1. Siller N, Kuhle J, Muthuraman M, et al. Serum neurofilament light chain is a biomarker of acute and chronic neuronal damage in early multiple sclerosis. Mult Scler 2019;25:678-686.

2. Kuhle J, Kropshofer H, Haering DA, et al. Blood neurofilament light chain as a biomarker of MS disease activity and treatment response. Neurology 2019;92: e1007-e1015.

3. Kuhle J, Plavina T, Barro C, et al. Neurofilament light levels are associated with longterm outcomes in multiple sclerosis. Mult Scler J 2019;26:1691-1699.

4. Lovelace MD, Varney B, Sundaram G, et al. Recent evidence for an expanded role of the kynurenine pathway of tryptophan metabolism in neurological diseases. Neuropharmacology 2017;112:373-388.

5. Fakan B, Szalardy L, Vecsei L. Exploiting the therapeutic potential of endogenous immunomodulatory systems in multiple sclerosis-special focus on the peroxisome proliferator-activated receptors (PPARs) and the kynurenines. Int J Mol Sci 2019;20: 426.

6. Lim CK, Bilgin A, Lovejoy DB, et al. Kynurenine pathway metabolomics predicts and provides mechanistic insight into multiple sclerosis progression. Sci Rep 2017;7: 41473.

7. Chatterjee P, Zetterberg H, Goozee K, et al. Plasma neurofilament light chain and amyloid-beta are associated with the kynurenine pathway metabolites in preclinical Alzheimer's disease. J Neuroinflammation 2019;16:186.

8. Rajda C, Galla Z, Polyak H, et al. Cerebrospinal fluid neurofilament light chain is associated with kynurenine pathway metabolite changes in multiple sclerosis. Int J Mol Sci 2020;21:2665.

9. Dalgas U, Langeskov-Christensen M, Stenager E, Riemenschneider M, Hvid LG. Exercise as medicine in multiple sclerosis-time for a paradigm shift: preventive, symptomatic, and disease-modifying aspects and perspectives. Curr Neurol Neurosci Rep 2019;19:88.

10. Campbell E, Coulter EH, Paul L. High intensity interval training for people with multiple sclerosis: a systematic review. Mult Scler Relat Disord 2018;24:55-63.

11. Joisten N, Proschinger S, Rademacher A, et al. High-intensity interval training reduces neutrophil-to-lymphocyte ratio in persons with multiple sclerosis during inpatient rehabilitation. Mult Scler 2020. doi: 10.1177/1352458520951382.

12. Zimmer P, Bloch $\mathrm{W}$, Schenk A, et al. High-intensity interval exercise improves cog nitive performance and reduces matrix metalloproteinases- 2 serum levels in person with multiple sclerosis: a randomized controlled trial. Mult Scler 2018;24:1635-1644.

13. Joisten N, Rademacher A, Bloch W, et al. Influence of different rehabilitative aerobic exercise programs on (anti-) inflammatory immune signalling, cognitive and functional capacity in persons with MS — study protocol of a randomized controlled trial. BMC Neurol 2019;19:37.

14. Langdon DW, Amato MP, Boringa J, et al. Recommendations for a Brief International Cognitive Assessment for Multiple Sclerosis (BICAMS). Mult Scler 2012;18 891-898.

15. Engel S, Friedrich M, Muthuraman M, et al. Intrathecal B-cell accumulation and axonal damage distinguish MRI-based benign from aggressive onset in MS. Neurol Neuroimmunol Neuroinflamm 2019;6:e595.

16. Bittner S, Steffen F, Uphaus T, et al. Clinical implications of serum neurofilament in newly diagnosed MS patients: a longitudinal multicentre cohort study. EBioMedicine 2020;56:102807.

17. Joisten N, Kummerhoff F, Koliamitra C, et al. Exercise and the Kynurenine pathway: current state of knowledge and results from a randomized cross-over study comparing acute effects of endurance and resistance training. Exerc Immunol Rev 2020;26: 24-42.

18. Henykova E, Vranova HP, Amakorova P, et al. Stable isotope dilution ultra-high performance liquid chromatography-tandem mass spectrometry quantitative profiling of tryptophan-related neuroactive substances in human serum and cerebrospinal fluid. J Chromatogr A 2016;1437:145-157.

19. Schwarcz R, Stone TW. The kynurenine pathway and the brain: challenges, controversies and promises. Neuropharmacology 2017;112:237-247.

20. Agudelo LZ, Femenia T, Orhan F, et al. Skeletal muscle PGC-1alphal modulates kynurenine metabolism and mediates resilience to stress-induced depression. Cell 2014;159:33-45.

21. DiNatale BC, Murray IA, Schroeder JC, et al. Kynurenic acid is a potent endogenous aryl hydrocarbon receptor ligand that synergistically induces interleukin-6 in the presence of inflammatory signaling. Toxicol Sci 2010;115:89-97.

22. Bansi J, Koliamitra C, Bloch W, et al. Persons with secondary progressive and relapsing remitting multiple sclerosis reveal different responses of tryptophan metabolism to acute endurance exercise and training. J Neuroimmunol 2018;314:101-105.

23. Lemos $\mathrm{H}$, Mohamed $\mathrm{E}, \mathrm{Ou} \mathrm{R}$, et al. Co-treatments to boost IDO activity and inhibit production of downstream catabolites induce durable suppression of experimental autoimmune encephalomyelitis. Front Immunol 2020;11:1256.

24. Baban B, Chandler PR, Sharma MD, et al. IDO activates regulatory T cells and blocks their conversion into Th17-like T cells. J Immunol 2009;183:2475-2483.

25. Jensen CS, Portelius E, Hogh P, et al. Effect of physical exercise on markers of neuronal dysfunction in cerebrospinal fluid in patients with Alzheimer's disease. Alzheimers Dement (N Y) 2017;3:284-290.

26. Bartlett DM, Govus A, Rankin $\mathrm{T}$, et al. The effects of multidisciplinary rehabilitation on neuroimaging, biological, cognitive and motor outcomes in individuals with premanifest Huntington's disease. J Neurol Sci 2020;416:117022. 


\section{Neurology $^{\odot}$ \\ Neuroimmunology \& Neuroinflammation}

\section{Exercise Diminishes Plasma Neurofilament Light Chain and Reroutes the Kynurenine Pathway in Multiple Sclerosis}

Niklas Joisten, Annette Rademacher, Clemens Warnke, et al.

Neurol Neuroimmunol Neuroinflamm 2021;8;

DOI 10.1212/NXI.0000000000000982

This information is current as of March 29, 2021

\section{Updated Information \& \\ Services}

References

Citations

Subspecialty Collections

Permissions \& Licensing

Reprints including high resolution figures, can be found at:

http://nn.neurology.org/content/8/3/e982.full.html

This article cites 26 articles, 2 of which you can access for free at: http://nn.neurology.org/content/8/3/e982.full.html\#\#ref-list-1

This article has been cited by 1 HighWire-hosted articles: http://nn.neurology.org/content/8/3/e982.full.html\#\#otherarticles

This article, along with others on similar topics, appears in the following collection(s):

Autoimmune diseases

http://nn.neurology.org//cgi/collection/autoimmune_diseases Multiple sclerosis

http://nn.neurology.org//cgi/collection/multiple_sclerosis

Information about reproducing this article in parts (figures,tables) or in its entirety can be found online at:

http://nn.neurology.org/misc/about.xhtml\#permissions

Information about ordering reprints can be found online:

http://nn.neurology.org/misc/addir.xhtml\#reprintsus

Neurol Neuroimmunol Neuroinflamm is an official journal of the American Academy of Neurology.

Published since April 2014, it is an open-access, online-only, continuous publication journal. Copyright

Copyright $\left({ }^{\circ} 2021\right.$ The Author(s). Published by Wolters Kluwer Health, Inc. on behalf of the American

Academy of Neurology.. All rights reserved. Online ISSN: 2332-7812.

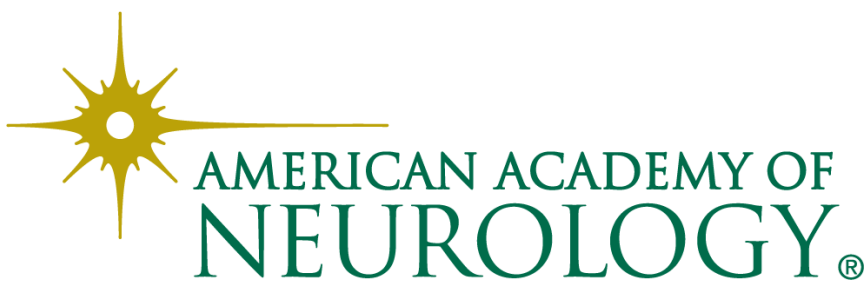

